\title{
Passivity Analysis of a System and its Approximation
}

\author{
M. Xia, P. J. Antsaklis and V. Gupta
}

\begin{abstract}
We consider the following problem: given two mathematical system models, one of which could represent accurately a physical system and the other an approximation of the system, what passivity properties of the system can be inferred from studying only the approximate model. Our results show that an excess of passivity (whether in the form of input strictly passive, output strictly passive or very strictly passive) in the approximate model guarantees a certain passivity index for the system, provided that the norm of the error between the two models is sufficiently small in a suitably defined sense. Further, we consider QSR dissipative systems and show that QSR dissipativity has a similar robustness property, even though the supply rates for the system and its approximation may be different.
\end{abstract}

\section{INTRODUCTION}

Energy dissipation is a fundamental concept in dynamical systems. Passivity and dissipativity characterize the "energy" consumption of a dynamical system and form a powerful tool in many real applications. Passivity is closely related to stability and exhibits a compositional property for parallel and feedback interconnections [1], [2], [3]. Passivity-based control is especially useful in the analysis of complex coupled systems.

In this paper, we are particularly interested in the passivity of a system as inferred from studying an approximate model of its dynamical behavior. In physical systems, precise knowledge of the mathematical model is difficult to obtain. Moreover, even if such a model were obtainable, the classical tradeoff between model accuracy and tractability may lead to the use of a simpler model [4]. A variety of approximation methods can be used, for analysis, simulation or control design of the 'real' systems [5]. While it is known that under some conditions, linearization [1], [6] and model reduction [7], [8] can be performed so as to preserve passivity, the question of whether passivity of a system can be guaranteed if a model 'close' to it is passive still remains open.

The main contribution of this paper is the establishment of relationships between passivity levels of two mathematical system models, one of which could represent accurately a physical system and the other could represent an approximation. Of course, the two mathematical models can represent two different approximations of the same physical system as well. The approximate model is assumed to have an excess of passivity, defined as passivity levels (similar to passivity indices [3]) that characterize how passive it is (how much of the energy introduced into the system is dissipated). If the

The authors are with the department of Electrical Engineering, University of Notre Dame, Notre Dame, IN 46556, USA. (e-mail: mxia, antsaklis.1, vgupta2@nd.edu). Research supported by the National Science Foundation under Grant No. CNS-1035655. error between the system and its approximation is "small" in a suitably defined sense, we show that the passivity levels for the system can be guaranteed. Since there is a rich theory of using passivity levels (or indices) to design controls [3], [9], our results imply that it is possible to use the (hopefully more tractable) approximate model for control design. An alternative interpretation of the results is as robustness properties with respect to model uncertainties [10], [11]. Further, if the approximate model does not have an excess of passivity, we assume that it is QSR dissipative and similar robustness properties can be derived. We apply our results to a particular approximation method: model reduction of a higher-order system to obtain a lower-order model which can be used to facilitate control designs or speed up simulations [5], [7]. Specifically, we consider linear systems and their positivereal truncations [8] and derive variations in the passivity levels for the full-order and reduced-order systems. The works such as [5], [7], [8] focus on how to preserve passivity. As opposed to these works, we show how passivity levels vary as a function of the order.

The rest of the paper is organized as follows. Section II provides background material on passivity and model reduction preserving passivity. Section III presents the problem statement. The main results are given in Section IV. Numerical examples are provided in Section V. Concluding remarks are given in Section VI.

Notation. The signal space under consideration is either the standard $\mathcal{L}_{2}$ space or the extended $\mathcal{L}_{2}$ space. The exact space will be clear from the context. The Euclidean space of dimension $m$ is denoted by $\mathbb{R}^{m}$. Denote the truncation of $u(t)$ up to time $T(0 \leq T<\infty)$ by $u_{T}(t)$. The inner product of truncated signals $u_{T}(t), y_{T}(t)$ is denoted by $\langle u, y\rangle_{T}$, where $\langle u, y\rangle_{T} \triangleq \int_{0}^{T} u^{T}(t) y(t) \mathrm{d} t$ and $u^{T}(t)$ denotes the transpose of $u(t)$. The $\mathcal{L}_{2}$-induced norm of the signal $u_{T}(t)$ is denoted by $\left\|u_{T}(t)\right\|_{\mathcal{L}_{2}}$, where $\left\|u_{T}(t)\right\|_{\mathcal{L}_{2}}^{2} \triangleq$ $\int_{0}^{T} u^{T}(t) u(t) \mathrm{d} t$. The $H_{\infty}$ norm of a transfer function $G(s)$ is denoted by $\|G\|_{H_{\infty}}$. For a matrix $A \in \mathbb{R}^{n \times n}$, the minimum eigenvalue of $A$ is denoted by $\underline{\lambda}(A)$ and the maximum eigenvalue by $\bar{\lambda}(A) \cdot \operatorname{Re}[A]$ is the real part of a complex matrix $A . A \geq 0$ denotes that $A$ is positive semi-definite and $A>0$ implies that $A$ is positive definite. The $n$ dimensional identity matrix is denoted by $I_{n \times n}$ or simply $I$ by omitting the dimensions if clear from the context. The notation $\max \{a, b\}$ denotes the larger value of $a, b \in \mathbb{R}$ and $\min \{a, b\}$ denotes the smaller value of $a, b \in \mathbb{R}$.

\section{PRELIMINARIES}

Definition 1 ([1], [12]): Consider a system $\Sigma$ with input $u$ and output $y$ where $u(t), y(t) \in \mathbb{R}^{m}$. It is said to be 
- passive, if there exists a constant $\beta \leq 0$ such that

$$
\langle u, y\rangle_{T} \geq \beta .
$$

- input strictly passive (ISP), if there exist constants $\nu>$ 0 and $\beta \leq 0$ such that

$$
\langle u, y\rangle_{T} \geq \beta+\nu\langle u, u\rangle_{T}
$$

- output strictly passive (OSP), if there exist constants $\rho>0$ and $\beta \leq 0$ such that

$$
\langle u, y\rangle_{T} \geq \beta+\rho\langle y, y\rangle_{T} .
$$

- very strictly passive (VSP), if there exist constants $\rho>$ $0, \nu>0$ and $\beta \leq 0$ such that

$$
\langle u, y\rangle_{T} \geq \beta+\rho\langle y, y\rangle_{T}+\nu\langle u, u\rangle_{T} .
$$

- finite-gain $\mathcal{L}_{2}$ stable, if there exist constants $\kappa>0$ and $\beta \leq 0$ such that

$$
\langle y, y\rangle_{T} \leq-\beta+\kappa^{2}\langle u, u\rangle_{T} .
$$

In all cases, the inequality should hold for $\forall u(t), \forall T \geq 0$ and the corresponding $y(t)$.

The constant $\beta$ is related to the initial condition of the system $\Sigma$ and plays an important role in the stability analysis of $\Sigma$ [12]. The inner product $\langle u, y\rangle_{T}$ may be interpreted as the externally supplied energy to $\Sigma$ during the interval $[0, T][1],[13]$. The above definitions can be viewed as special cases of QSR-dissipative systems [2], [14], defined as systems for which there exist $Q=Q^{T}, R=R^{T}$ and $S$, such that $\forall u(t), \forall T \geq 0$ and the corresponding $y(t)$,

$$
r(u, y) \triangleq\langle y, Q y\rangle_{T}+2\langle y, S u\rangle_{T}+\langle u, R u\rangle_{T} \geq 0 .
$$

The function $r(u, y)$ is called the supply rate for $\Sigma$.

If a system $\Sigma$ is ISP for $\nu>0$, it is also ISP for $\nu-\epsilon$, where $0 \leq \epsilon<\nu$. Analogously, if $\Sigma$ is OSP for $\rho>0$, it is also OSP for $\rho-\epsilon$, where $0 \leq \epsilon<\rho$ [3]. Finally, if $\Sigma$ is VSP for $(\rho, \nu)$, i.e. (3) holds, it is also VSP for $(\rho-\epsilon, \nu-\epsilon)$, where $0 \leq \epsilon<\min \{\rho, \nu\}$ (see [15] for a complete proof). A positive value of $\rho$ or $\nu$ can thus be interpreted as an excess of passivity and these two values (called passivity levels) characterize 'how passive' $\Sigma$ is. If $\rho$ or $\nu$ is negative, we say $\Sigma$ has a shortage of passivity. This intuition is captured by the concept of passivity indices [3].

Definition 2: For a system $\Sigma$ with input $u$ and output $y$ where $u(t), y(t) \in \mathbb{R}^{m}$,

- the input feedforward passivity index (IFP) is the largest $\nu>0$ such that (1) holds for $\forall u$ and $\forall T \geq 0$,

- the output feedback passivity index (OFP) is the largest $\rho>0$ such that (2) holds for $\forall u$ and $\forall T \geq 0$.

The two indices are denoted by $\operatorname{IFP}(\nu)$ and $\operatorname{OFP}(\rho)$, respectively.

Note the fact that a system has $\operatorname{IFP}(\nu)$ and $\operatorname{OFP}(\rho)$ does not necessarily imply that the system is VSP for $(\rho, \nu)$. In other words, (3) may not hold for the passivity indices $\rho$ and $\nu$. A necessary condition for $\rho$ and $\nu$ to be VSP is given by $\rho \nu \leq \frac{1}{4}, \rho>0, \nu>0$ (see [15] for a complete proof). As a result, for VSP, it may not make sense to define the largest $\rho>0$ and the largest $\nu>0$ (simultaneously) such that (3) holds for $\forall u$ and $\forall T \geq 0$. We thus use the notion of passivity levels. Consider the system $\Sigma$,

- any $\tilde{\nu} \in(0, \nu]$ is a passivity level of $\Sigma$ if $\Sigma$ has $\operatorname{IFP}(\nu)$;

- any $\tilde{\rho} \in(0, \rho]$ is a passivity level of $\Sigma$ if $\Sigma$ has $\operatorname{OFP}(\rho)$;

- any $(\tilde{\rho}, \tilde{\nu})$ are passivity levels of $\Sigma$ if $\Sigma$ is $\operatorname{VSP}$ for $(\rho, \nu)$ such that $0<\tilde{\rho} \leq \rho, 0<\tilde{\nu} \leq \nu$.

Consider a linear time-invariant system with transfer function $G(s)$, a minimal state-space realization is given by

$$
\begin{aligned}
& \dot{x}=A x+B u, \\
& y=C x+D u,
\end{aligned}
$$

where $\{A, B\}$ is controllable and $\{A, C\}$ is observable. The following result is useful to test whether system (6) is passive.

Lemma 1 ([13]): System (6) is passive if and only if there exist matrices $P=P^{T}>0, L$ and $W$, such that

$$
\begin{aligned}
P A+A^{T} P & =-L^{T} L, \\
P B & =C^{T}-L^{T} W, \\
W^{T} W & =D+D^{T} .
\end{aligned}
$$

A special case of system (6) is of relaxation type, i.e.

$$
A=A^{T}, A \leq 0, B^{T}=C, D \geq 0 .
$$

Relaxed systems play an important role in applications and examples of such systems include integrated circuits and mechanical systems where inertial effects may be neglected, see e.g. [8], [14].

The algorithm for model reduction considered in this paper preserves passivity, called positive-real truncated balancing realization (PR-TBR for short), as presented in [8]. The observability grammian $W_{o}$ and the controllability grammian $W_{c}$ of system (6) can be used as a basis for PR-TBR procedure when (8) is satisfied. The square roots of the eigenvalues of the product $W_{c} W_{o}$ are called Hankel singular values and can be used to establish upper bounds on the difference between the transfer function of the full-order system $G_{1}$ and its reduced order approximation $G_{2}$. If we denote $\sigma_{i}$ as the $i$ th Hankel singular value (where $\sigma_{1} \geq \sigma_{2} \geq$ $\cdots \geq \sigma_{n}$ and $n$ is the order of $G_{1}$ ), then

$$
\left\|G_{1}-G_{2}\right\|_{H_{\infty}} \leq 2 \sum_{i=r+1}^{n} \sigma_{i},
$$

where $r$ is the order of the reduced order model $G_{2}$ [5], [7].

Remark 1: There exist various methods for model reduction (of linear or nonlinear systems), but we do not concentrate on that problem. Linear models of relaxation type and model reduction preserving passivity are used merely as illustrating examples of our main results.

\section{Problem Statement}

Consider two system models $\Sigma_{1}$ and $\Sigma_{2}$ as shown in Fig. 1. One can view $\Sigma_{i}$ as the system we are interested in and $\Sigma_{j}$ as an approximation of $\Sigma_{i}$, where $i, j \in\{1,2\}$ and $j \neq i$. A commonly used measure for judging how well $\Sigma_{j}$ approximates $\Sigma_{i}$ is to compare the outputs for the same 


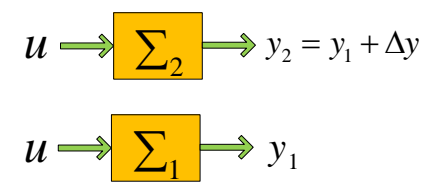

Fig. 1. Illustration of two systems: $\Sigma_{1}$ with input $u$ and output $y_{1}$ and $\Sigma_{2}$ with input $u$ and output $y_{2}=y_{1}+\Delta y$, where $u, y_{1}, y_{2}$ and $\Delta y$ are of the same dimensions.

excitation function $u$ [5]. We denote the difference in the outputs by $\Delta y$. Note that in general $\Delta y$ will depend on the exact function $u$. The error may be due to modeling, linearization or model reduction or a host of other reasons. For a 'good' approximation, we require that the "worst" case $\Delta y$ over all control inputs $u$ be small. More formally, we say that $\Sigma_{j}$ is a good approximation of $\Sigma_{i}$ if there exists a positive constant $\gamma>0$ such that

$$
\langle\Delta y, \Delta y\rangle_{T} \leq \gamma^{2}\langle u, u\rangle_{T}, \quad \forall u \text { and } \forall T \geq 0 .
$$

The value of $\gamma$ obviously reflects how good the approximation is. In the following analysis, without loss of generality, we view $\Sigma_{2}$ as an approximation of $\Sigma_{1}$.

Remark 2: Note that (10) actually requires the 'error system' with input $u$ and output $\Delta y$ to be $\mathcal{L}_{2}$ stable. For stable linear systems with zero initial conditions, $\Sigma_{1}$ (resp. $\Sigma_{2}$ ) is characterized by the transfer function $G_{1}$ (resp. $G_{2}$ ). Defining $\Delta G=G_{1}-G_{2}$, if $\|\Delta G\|_{H_{\infty}} \leq \gamma$, (10) is satisfied. Thus, $\gamma$ is an upper bound on the $H_{\infty}$ norm of the difference in the transfer functions $G_{1}$ and $G_{2}$. In particular, if $G_{2}$ is a reduced order model of $G_{1}$ that obtained through the PRTBR procedure, from (9), we obtain that the 'error' constraint $\gamma$ in (10) can be calculated as $2 \sum_{i=r+1}^{n} \sigma_{i}$.

We are now ready to state the problem of interest. Assume that $\Sigma_{2}$ has an excess of passivity, namely $\Sigma_{2}$ has $\operatorname{IFP}(\nu)$ or $\operatorname{OFP}(\rho)$ or is $\operatorname{VSP}$ for $(\rho, \nu)$. What passivity property for $\Sigma_{1}$ can be inferred from that of $\Sigma_{2}$ ? For the case when $\Sigma_{2}$ does not have an excess of passivity, we assume it to be $\left(Q_{2}, S_{2}, R_{2}\right)$-dissipative and consider the problem of obtaining conditions under which $\Sigma_{1}$ is $\left(Q_{1}, S_{1}, R_{1}\right)$ dissipative as well. The problem is summarized as follows.

Problem 1: Suppose that an approximate model $\Sigma_{2}$

1) has $\operatorname{IFP}(\nu)$; or

2) has $\operatorname{OFP}(\rho)$; or

3) is VSP for $(\rho, \nu)$; or

4) is $\left(Q_{2}, S_{2}, R_{2}\right)$-dissipative.

What corresponding passivity or QSR-dissipativity properties can be derived for the system $\Sigma_{1}$ based on (10)?

\section{MAin Results}

We begin by considering the case when the approximate model is ISP and then move on to the cases when the approximation is OSP, VSP or QSR-dissipative, subsequently.

\section{A. Input Strictly Passive Systems}

We have the following result that guarantees a certain passivity level given the error constraint $\gamma$ and IFP of the approximate model.
Theorem 1: Consider $\Sigma_{1}$ and $\Sigma_{2}$ in Fig. 1. Suppose (10) is satisfied for some $\gamma>0$. If $\Sigma_{2}$ has $\operatorname{IFP}(\nu)$ and $\gamma<\nu$, then $\Sigma_{1}$ is ISP for $\tilde{\nu}=\nu-\gamma$.

Proof: From Cauchy-Schwarz inequality and the assumption (10), we obtain

$$
\left|\langle u, \Delta y\rangle_{T}\right| \leq \sqrt{\langle u, u\rangle_{T}} \sqrt{\langle\Delta y, \Delta y\rangle_{T}} \leq \gamma\langle u, u\rangle_{T},
$$

For the system $\Sigma_{2}$ with input $u$ and output $y_{2}$, we have

$$
\begin{aligned}
& \left\langle u, y_{2}\right\rangle_{T}-\nu\langle u, u\rangle_{T} \\
= & \left\langle u, y_{1}\right\rangle_{T}-\nu\langle u, u\rangle_{T}+\langle u, \Delta y\rangle_{T} \\
\leq & \left\langle u, y_{1}\right\rangle_{T}-\nu\langle u, u\rangle_{T}+\left|\langle u, \Delta y\rangle_{T}\right| \\
\leq & \left\langle u, y_{1}\right\rangle_{T}-(\nu-\gamma)\langle u, u\rangle_{T} .
\end{aligned}
$$

Now, by assumption, $\Sigma_{2}$ is ISP for $\nu>0$, then

$$
\left\langle u, y_{2}\right\rangle_{T}-\nu\langle u, u\rangle_{T} \geq \beta .
$$

Therefore, defining $\tilde{\nu}=\nu-\gamma>0$, we obtain $\left\langle u, y_{1}\right\rangle_{T}-$ $\tilde{\nu}\langle u, u\rangle_{T} \geq \beta$. This implies $\Sigma_{1}$ is ISP for $\tilde{\nu}>0$.

Note $\tilde{\nu}$ does not represent the IFP of $\Sigma_{1}\left(\Sigma_{1}\right.$ may have IFP larger than $\tilde{\nu}$ ). If we are merely interested in determining whether $\Sigma_{1}$ is passive (rather than characterizing the passivity level of $\Sigma_{1}$ ), we can allow $\gamma$ to be equal to $\nu$.

Corollary 1: Consider $\Sigma_{1}$ and $\Sigma_{2}$ in Fig. 1. Suppose (10) is satisfied for some $\gamma>0$. If $\Sigma_{2}$ has $\operatorname{IFP}(\nu)$ and $\gamma \leq \nu$, then, $\Sigma_{1}$ is passive.

Proof: From (11) and $\gamma \leq \nu$, we obtain

$$
\left|\langle u, \Delta y\rangle_{T}\right| \leq \gamma\langle u, u\rangle_{T} \leq \nu\langle u, u\rangle_{T}
$$

The following relation holds for $\Sigma_{1}$

$$
\begin{aligned}
\left\langle u, y_{1}\right\rangle_{T} & =\left\langle u, y_{2}\right\rangle_{T}-\langle u, \Delta y\rangle_{T} \\
& \geq\left\langle u, y_{2}\right\rangle_{T}-\left|\langle u, \Delta y\rangle_{T}\right| \\
& \geq\left\langle u, y_{2}\right\rangle_{T}-\nu\langle u, u\rangle_{T} \geq \beta .
\end{aligned}
$$

Therefore, $\left\langle u, y_{1}\right\rangle_{T} \geq \beta$, i.e. $\Sigma_{1}$ is passive.

\section{B. Output Strictly Passive Systems}

For OSP systems, we assume along the lines of [3] that the inverse of $\Sigma_{2}$ is causal and $\mathcal{L}_{2}$ stable. For linear system (6), a necessary condition to satisfy this assumption is that $G(s)$ has relative degree zero and is minimum phase, i.e. all the zeros of $G(s)$ have negative real parts. In this case, the OFP for $G(s)$ is shown to be equivalent to the IFP of the inverse of $G(s)$, see e.g. [3].

Assumption 1: Consider $\Sigma_{2}$ with input $u$ and output $y_{2}$. Assume the inverse of $\Sigma_{2}$ is causal and stable, i.e. there exist $\eta>0$, such that $\forall y_{2}, \forall T \geq 0$

$$
\langle u, u\rangle_{T} \leq \eta^{2}\left\langle y_{2}, y_{2}\right\rangle_{T}
$$

With this assumption, we have the following result.

Theorem 2: Consider $\Sigma_{1}$ and $\Sigma_{2}$ in Fig. 1. Suppose (10) holds for some $\gamma>0$ and (12) holds for some $\eta>0$. If $\Sigma_{2}$ has $\operatorname{OFP}(\rho)$ and $\gamma<\rho$, then $\Sigma_{1}$ is OSP for $\tilde{\rho}=\rho-\gamma$ if

$$
\frac{1}{\eta^{2}}-\left(1+2(\rho-\gamma) \frac{1}{\rho}+(\rho-\gamma) \gamma\right) \geq 0 \text {. }
$$


Proof: For all $\rho>0$, we have the following relation $u^{T} y_{2} \leq \frac{1}{2 \rho} u^{T} u+\frac{\rho}{2} y_{2}^{T} y_{2}$ and thus

$$
u^{T} y_{2}-\rho y_{2}^{T} y_{2} \leq \frac{1}{2 \rho} u^{T} u-\frac{\rho}{2} y_{2}^{T} y_{2}
$$

$\Sigma_{2}$ is assumed to be OSP for $\rho>0$, thus

$$
\frac{1}{2 \rho}\langle u, u\rangle_{T}-\frac{\rho}{2}\left\langle y_{2}, y_{2}\right\rangle_{T} \geq\left\langle u, y_{2}\right\rangle_{T}-\rho\left\langle y_{2}, y_{2}\right\rangle_{T} \geq \beta,
$$

and therefore $\left\langle y_{2}, y_{2}\right\rangle_{T} \leq \frac{1}{\rho^{2}}\langle u, u\rangle_{T}-\frac{2 \beta}{\rho}$. From CauchySchwarz inequality, (10) and the fact $\beta \leq 0$, we obtain

$$
\begin{aligned}
\left|\left\langle y_{2}, \Delta y\right\rangle_{T}\right| & \leq \sqrt{\langle\Delta y, \Delta y\rangle_{T}} \sqrt{\left\langle y_{2}, y_{2}\right\rangle_{T}} \\
& \leq \frac{\gamma}{\rho} \sqrt{\langle u, u\rangle_{T}} \sqrt{\langle u, u\rangle_{T}-2 \beta \rho} \\
& \leq \frac{\gamma}{\rho}\left(\langle u, u\rangle_{T}-2 \beta \rho\right)=\frac{\gamma}{\rho}\langle u, u\rangle_{T}-2 \beta \gamma
\end{aligned}
$$

Together with (11), if we define $a \triangleq \rho-\gamma>0$, we obtain

$$
\begin{aligned}
\Phi & \triangleq \gamma\left\langle y_{2}, y_{2}\right\rangle_{T}-\langle u, \Delta y\rangle_{T}+2 a\left\langle\Delta y, y_{2}\right\rangle_{T}-a\langle\Delta y, \Delta y\rangle_{T} \\
& \geq \gamma\left\langle y_{2}, y_{2}\right\rangle_{T}-\left|\langle u, \Delta y\rangle_{T}\right|-2 a\left|\left\langle\Delta y, y_{2}\right\rangle_{T}\right|-a \gamma^{2}\langle u, u\rangle_{T} \\
& \geq \gamma\left\langle y_{2}, y_{2}\right\rangle_{T}-\left(\gamma+2 a \frac{\gamma}{\rho}+a \gamma^{2}\right)\langle u, u\rangle_{T}+4 a \beta \gamma
\end{aligned}
$$

If (13) is satisfied, from assumption (12), we obtain

$$
\begin{aligned}
& \gamma\left\langle y_{2}, y_{2}\right\rangle_{T}-\left(\gamma+2 a \frac{\gamma}{\rho}+a \gamma^{2}\right)\langle u, u\rangle_{T} \\
& \geq\left[\frac{1}{\eta^{2}}-\left(1+2 a \frac{1}{\rho}+a \gamma\right)\right] \gamma \eta^{2}\left\langle y_{2}, y_{2}\right\rangle_{T} \geq 0
\end{aligned}
$$

Thus, $\Phi \geq 4 a \beta \gamma$. For $\Sigma_{1}$ with $y_{1}=y_{2}-\Delta y$,

$$
\begin{aligned}
& \left\langle u, y_{1}\right\rangle_{T}-(\rho-\gamma)\left\langle y_{1}, y_{1}\right\rangle_{T} \\
= & \left\langle u, y_{2}\right\rangle_{T}-\rho\left\langle y_{2}, y_{2}\right\rangle_{T}+\Phi \geq \beta+4 a \beta \gamma \triangleq \bar{\beta},
\end{aligned}
$$

for all functions $u$, all $T \geq 0$ and $\bar{\beta} \leq 0$. Therefore, for $\gamma<\rho, \Sigma_{1}$ is OSP for $\tilde{\rho}=\rho-\gamma$.

Note that $\Sigma_{1}$ may have OFP larger than $\tilde{\rho}$. If we are merely interested in passivity of $\Sigma_{1}$, we have the following result.

Corollary 2: Consider $\Sigma_{1}$ and $\Sigma_{2}$ in Fig. 1. Suppose (10) holds for some $\gamma>0$ and (12) holds for some $\eta>0$. If $\Sigma_{2}$ has $\operatorname{OFP}(\rho)$ and $\gamma \eta^{2} \leq \rho$, then, $\Sigma_{1}$ is passive.

Proof: From (11) and the assumption (12), we obtain

$$
\left|\langle u, \Delta y\rangle_{T}\right| \leq \gamma\langle u, u\rangle_{T} \leq \gamma \eta^{2}\left\langle y_{2}, y_{2}\right\rangle_{T}
$$

Thus, the following relation holds if $\gamma \eta^{2} \leq \rho$,

$$
\begin{aligned}
\left\langle u, y_{1}\right\rangle_{T} & =\left\langle u, y_{2}\right\rangle_{T}-\langle u, \Delta y\rangle_{T} \\
& \geq\left\langle u, y_{2}\right\rangle_{T}-\rho\left\langle y_{2}, y_{2}\right\rangle_{T}-\left|\langle u, \Delta y\rangle_{T}\right|+\rho\left\langle y_{2}, y_{2}\right\rangle_{T} \\
& \geq \beta+\left(\rho-\gamma \eta^{2}\right)\left\langle y_{2}, y_{2}\right\rangle_{T} \geq \beta .
\end{aligned}
$$

Therefore, $\left\langle u, y_{1}\right\rangle_{T} \geq \beta$, i.e. $\Sigma_{1}$ is passive.

\section{Very Strictly Passive Systems}

We have the following result.

Theorem 3: Consider $\Sigma_{1}$ and $\Sigma_{2}$ in Fig. 1. Suppose (10) holds for some $\gamma>0$. Suppose $\Sigma_{2}$ is $\operatorname{VSP}$ for $(\rho, \nu)$, where $\rho>\gamma, \nu>\gamma$. Then, $\Sigma_{1}$ is VSP for $(\rho-\gamma, \nu-\gamma)$ if

$$
\nu^{2}-\frac{2(\rho-\gamma)}{\rho}-(\rho-\gamma) \gamma \geq 0 \text {. }
$$

Proof: We use the relation $u^{T} y_{2}-\nu u^{T} u \leq \frac{1}{2 \nu} y_{2}^{T} y_{2}-$ $\frac{\nu}{2} u^{T} u . \Sigma_{2}$ is assumed to be ISP for $\nu>0$, thus

$$
\frac{1}{2 \nu}\left\langle y_{2}, y_{2}\right\rangle_{T}-\frac{\nu}{2}\langle u, u\rangle_{T} \geq\left\langle u, y_{2}\right\rangle_{T}-\nu\langle u, u\rangle_{T} \geq \beta,
$$

and therefore $\left\langle y_{2}, y_{2}\right\rangle_{T} \geq \nu^{2}\langle u, u\rangle_{T}+2 \beta \nu$. Also, $\Sigma_{2}$ is OSP for $\rho>0$, thus (14) is satisfied. Together with (10) and (11), if we define $a=\rho-\gamma>0, \psi=2 a\left\langle y_{2}, \Delta y\right\rangle_{T}-\langle u, \Delta y\rangle_{T}-$ $a\langle\Delta y, \Delta y\rangle_{T}$, we obtain

$$
\begin{aligned}
|\psi| & \leq\left|\langle u, \Delta y\rangle_{T}\right|+2 a\left|\left\langle y_{2}, \Delta y\right\rangle_{T}\right|+a\langle\Delta y, \Delta y\rangle_{T} \\
& \leq\left(\gamma+2 a \frac{\gamma}{\rho}+a \gamma^{2}\right)\langle u, u\rangle_{T}-4 a \beta \gamma .
\end{aligned}
$$

Thus, the following relation holds

$$
\begin{aligned}
& \gamma\langle u, u\rangle_{T}+\gamma\left\langle y_{2}, y_{2}\right\rangle_{T}+\psi \\
\geq & \gamma\left(1+\nu^{2}\right)\langle u, u\rangle_{T}+2 \beta \nu \gamma-|\psi| \\
\geq & {\left[\gamma\left(1+\nu^{2}\right)-\left(\gamma+2 a \frac{\gamma}{\rho}+a \gamma^{2}\right)\right]\langle u, u\rangle_{T}+2 \beta \nu \gamma+4 a \beta \gamma } \\
= & \gamma\left(\nu^{2}-\frac{2 a}{\rho}-a \gamma\right)\langle u, u\rangle_{T}+2 \beta \nu \gamma+4 a \beta \gamma .
\end{aligned}
$$

We assume that $\nu^{2}-\frac{2 a}{\rho}-a \gamma \geq 0$ from (15), thus

$$
\gamma\langle u, u\rangle_{T}+\gamma\left\langle y_{2}, y_{2}\right\rangle_{T}+\psi \geq 2 \beta \nu \gamma+4 a \beta \gamma
$$

For $\Sigma_{1}$ with input $u$ and output $y_{1}=y_{2}-\Delta y$, we have

$$
\begin{aligned}
& \left\langle u, y_{1}\right\rangle_{T}-(\nu-\gamma)\langle u, u\rangle_{T}-(\rho-\gamma)\left\langle y_{1}, y_{1}\right\rangle_{T} \\
= & \left\langle u, y_{2}\right\rangle_{T}-\nu\langle u, u\rangle_{T}-\rho\left\langle y_{2}, y_{2}\right\rangle_{T} \\
& +\gamma\langle u, u\rangle_{T}+\gamma\left\langle y_{2}, y_{2}\right\rangle_{T}+\psi \\
\geq & \left\langle u, y_{2}\right\rangle_{T}-\nu\langle u, u\rangle_{T}-\rho\left\langle y_{2}, y_{2}\right\rangle_{T}+2 \beta \nu \gamma+4 a \beta \gamma .
\end{aligned}
$$

$\Sigma_{2}$ is assumed to be VSP for $(\rho, \nu)$ and therefore

$$
\left\langle u, y_{2}\right\rangle_{T}-\nu\langle u, u\rangle_{T}-\rho\left\langle y_{2}, y_{2}\right\rangle_{T} \geq \beta .
$$

Defining $\bar{\beta}=\beta+2 \beta \nu \gamma+4 a \beta \gamma \leq 0$, we have

$$
\left\langle u, y_{1}\right\rangle_{T}-(\nu-\gamma)\langle u, u\rangle_{T}-(\rho-\gamma)\left\langle y_{1}, y_{1}\right\rangle_{T} \geq \bar{\beta} .
$$

Thus, for $\gamma<\rho, \gamma<\nu, \Sigma_{1}$ is VSP for $(\rho-\gamma, \nu-\gamma)$.

$\Sigma_{1}$ is VSP for $(\rho, \nu)$ implies that $\rho$ is a passivity level for OSP and $\nu$ is a passivity level for ISP. The OFP of $\Sigma_{1}$ is larger than $\rho$ and the IFP is larger than $\nu$ in general.

Corollary 3: Consider $\Sigma_{1}$ and $\Sigma_{2}$ in Fig. 1. Suppose (10) holds for some $\gamma>0$. If $\Sigma_{2}$ is VSP for $(\rho, \nu)$ and $\rho \nu^{2}+$ $\nu-\gamma \geq 0$, then, $\Sigma_{1}$ is passive.

Proof: $\quad \Sigma_{2}$ is ISP for $\nu$, it has been shown that $\left\langle y_{2}, y_{2}\right\rangle_{T} \geq \nu^{2}\langle u, u\rangle_{T}+2 \beta \nu$. From (11), we obtain

$$
\begin{aligned}
\chi \triangleq & -\left|\langle u, \Delta y\rangle_{T}\right|+\rho\left\langle y_{2}, y_{2}\right\rangle_{T}+\nu\langle u, u\rangle_{T} \\
& \geq\left(\rho \nu^{2}+\nu-\gamma\right)\langle u, u\rangle_{T}+2 \beta \rho \nu .
\end{aligned}
$$


Thus, if $\rho \nu^{2}+\nu-\gamma \geq 0$, we obtain $\chi \geq 2 \beta \rho \nu$. $\Sigma_{2}$ is VSP for $(\rho, \nu)$, thus $\left\langle u, y_{2}\right\rangle_{T}-\rho\left\langle y_{2}, y_{2}\right\rangle_{T}-\nu\langle u, u\rangle_{T} \geq \beta$. For $\Sigma_{1}$ with input $u$ and output $y_{1}$, we have

$$
\begin{aligned}
\left\langle u, y_{1}\right\rangle_{T} & =\left\langle u, y_{2}\right\rangle_{T}-\langle u, \Delta y\rangle_{T} \\
& \geq\left\langle u, y_{2}\right\rangle_{T}-\rho\left\langle y_{2}, y_{2}\right\rangle_{T}-\nu\langle u, u\rangle_{T}+\chi \\
& \geq \beta+2 \beta \rho \nu \triangleq \bar{\beta}
\end{aligned}
$$

Thus, $\left\langle u, y_{1}\right\rangle_{T} \geq \bar{\beta}$ and $\bar{\beta} \leq 0$, i.e. $\Sigma_{1}$ is passive.

Remark 3: It can be verified that the above results hold when $\Sigma_{1}$ and $\Sigma_{2}$ exchange places. In other words, it does not really matter whether we view $\Sigma_{1}$ as an approximation of $\Sigma_{2}$ or $\Sigma_{2}$ as an approximation of $\Sigma_{1}$. In practice, however, a simple model is usually used as an approximation of a complex system, e.g. linearized model vs. nonlinear model and lower-order model vs. higher-order model.

Remark 4: Theorem 1-3 relate passivity levels between $\Sigma_{1}$ and $\Sigma_{2}$ for ISP, OSP and VSP systems. It is worth stressing that these results are applicable to any approximation methods and any system structure in general. In particular, if we consider linear systems and PR-TBR as a particular approximation approach, then the results in Theorem 1-3 provide a tool to trade off the 'error' constraint $\gamma$ in (10) as a function of variations in the passivity levels for the fullorder system $\Sigma_{1}$ (or $\Sigma_{2}$ ) and the reduced-order system $\Sigma_{2}$ (or $\Sigma_{1}$ ) (see [15] for more details).

\section{Extension to QSR-dissipative Systems}

In this section, we extend the results to QSR-dissipative systems, for which the system may be not passive or have a shortage of passivity.

Theorem 4: Consider $\Sigma_{1}$ and $\Sigma_{2}$ in Fig. 1. Suppose (10) holds for some $\gamma>0$. Let $\Sigma_{2}$ be $\left(Q_{2}, S_{2}, R_{2}\right)$-dissipative and assume $S_{1}-S_{2}=0, Q_{1}-Q_{2}>0, R_{1}-R_{2}>0$. If there exists a constant $\xi>0$ such that

$$
\begin{aligned}
\underline{\lambda}\left(R_{1}-R_{2}\right)-\frac{\gamma^{2}}{\xi}-2 \lambda_{1} \gamma-b & \geq 0, \\
\underline{\lambda}\left(Q_{1}-Q_{2}\right)-\xi \lambda_{2} & \geq 0,
\end{aligned}
$$

where $b=2 \max \left\{0, \bar{\lambda}\left(-Q_{1}\right) \gamma^{2}\right\}$, and

$$
\lambda_{1} \triangleq \sqrt{\bar{\lambda}\left(S_{1}^{T} S_{1}\right)} \geq 0, \lambda_{2} \triangleq \bar{\lambda}\left(Q_{1}^{T} Q_{1}\right) \geq 0
$$

then $\Sigma_{1}$ is $\left(Q_{1}, S_{1}, R_{1}\right)$-dissipative.

Proof: From Cauchy-Schwarz inequality, we obtain

$$
\left|\left\langle S_{1} u, \Delta y\right\rangle_{T}\right| \leq \sqrt{\bar{\lambda}\left(S_{1}^{T} S_{1}\right)} \gamma\langle u, u\rangle_{T}=\lambda_{1} \gamma\langle u, u\rangle_{T}
$$

Also, for some $\xi>0$, the following relation holds

$$
2\left\langle Q_{1} y_{2}, \Delta y\right\rangle_{T} \leq \frac{\gamma^{2}}{\xi}\langle u, u\rangle_{T}+\xi \lambda_{2}\left\langle y_{2}, y_{2}\right\rangle_{T}
$$

Define the supply rate for $\Sigma_{i}$ as $r_{i}\left(u, y_{i}\right) \triangleq\left\langle y_{i}, Q_{i} y_{i}\right\rangle_{T}+$ $2\left\langle y_{i}, S_{i} u\right\rangle_{T}+\left\langle u, R_{i} u\right\rangle_{T}$, then

$$
\begin{aligned}
r_{1}= & r_{2}+\left\langle y_{2},\left(Q_{1}-Q_{2}\right) y_{2}\right\rangle_{T}+\left\langle u,\left(R_{1}-R_{2}\right) u\right\rangle_{T} \\
& -2\left\langle y_{2}, Q_{1} \Delta y\right\rangle_{T}+\left\langle\Delta y, Q_{1} \Delta y\right\rangle_{T}-2\left\langle\Delta y, S_{1} u\right\rangle_{T} \\
\geq & r_{2}+\left(\underline{\lambda}\left(Q_{1}-Q_{2}\right)-\xi \lambda_{2}\right)\left\langle y_{2}, y_{2}\right\rangle_{T}+\left\langle\Delta y, Q_{1} \Delta y\right\rangle_{T} \\
& +\left(\underline{\lambda}\left(R_{1}-R_{2}\right)-\frac{\gamma^{2}}{\xi}-2 \lambda_{1} \gamma\right)\langle u, u\rangle_{T} .
\end{aligned}
$$

Since $\Sigma_{2}$ is $\left(Q_{2}, S_{2}, R_{2}\right)$-dissipative, $r_{2} \geq 0$. Two cases are possible. If $Q_{1}>0$, we have $b=0,\left\langle\Delta y, Q_{1} \Delta y\right\rangle_{T} \geq 0$. Thus, from (16), we obtain $r_{1} \geq r_{2} \geq 0$. If $Q_{1} \leq 0$, we have $b=\bar{\lambda}\left(-Q_{1}\right) \gamma^{2}$ and from (10),

$$
\left\langle\Delta y, Q_{1} \Delta y\right\rangle_{T} \geq-\bar{\lambda}\left(-Q_{1}\right) \gamma^{2}\langle u, u\rangle_{T} .
$$

If (16) holds, we obtain $r_{1} \geq r_{2} \geq 0$. In summary, $r_{1} \geq 0$ if (16) is satisfied and thus $\Sigma_{1}$ is $\left(Q_{1}, S_{1}, R_{1}\right)$-dissipative.

Remark 5: Similar arguments can be developed when $S_{1}-S_{2}=0, Q_{1}-Q_{2}>0, R_{1}-R_{2}>0$ does not hold. However, the analysis is more involved.

\section{NumERICAL EXAMPLES}

In this section, we provide a few numerical examples to illustrate the theoretical results developed in this paper. In general, $\Sigma_{1}$ represents the system we are interested in and and $\Sigma_{2}$ is an approximation of $\Sigma_{1}$. We assume zero initial conditions. For linear systems, the behavior of system $\Sigma_{i}$ is determined by the corresponding transfer function $G_{i}$. The approximation method we consider here is the PR-TBR procedure for model reduction (see e.g. [8]) and the system models are of relaxation type.

Example 1: The original system $G_{1}$ given by (17) is of order 8 . The Hankel singular values are given by $\Lambda$ in (18) and ordered as $\sigma_{1} \geq \cdots \geq \sigma_{8}$. Its second-order approximation is given by

$$
G_{2}=\frac{0.5 s^{2}+21.96 s+47.85}{s^{2}+5.8 s+4.456}
$$

and the $\operatorname{IFP}(\nu)$ for $G_{2}$ (defined in [3]) can be computed as

$$
\nu=\min _{w \in \mathbb{R}} \operatorname{Re}\left[G_{2}(j w)\right]=0.5 .
$$

The error in the transfer functions is given as [8]

$$
\left\|G_{1}-G_{2}\right\|_{H_{\infty}} \leq 2 \sum_{k=3}^{8} \sigma_{k}=0.0803
$$

Thus, $\gamma=0.0803<0.5$. According to Theorem $1, G_{1}$ is ISP for $\tilde{\rho}=\rho-\gamma=0.5-0.0803=0.4197$. This is true because the IFP for $G_{1}$ is actually 0.5 , larger than $\tilde{\rho}=0.4197$.

Example 2: The original system $G_{1}$ is given by

$$
\frac{1.8 s^{5}+53.56 s^{4}+590.8 s^{3}+3034 s^{2}+7279 s+6543}{s^{5}+23 s^{4}+203.1 s^{3}+861.7 s^{2}+1759 s+1382} .
$$

Its first-order approximation is given by

$$
G_{2}=\frac{1.8 s+19.37}{s+4.132}
$$




$$
\begin{aligned}
& G_{1}= \frac{0.5 s^{8}+28.6 s^{7}+352.2 s^{6}+1887 s^{5}+5299 s^{4}+8295 s^{3}+7190 s^{2}+3173 s+542.9}{s^{8}+18.5 s^{7}+133.5 s^{6}+496.1 s^{5}+1047 s^{4}+1290 s^{3}+911.1 s^{2}+337.5 s+50.18} \\
& \Lambda=\operatorname{diag}\left\{4.6357,0.4834,0.0375,0.0023,3.5 \times 10^{-4}, 1.9 \times 10^{-5}, 0,0\right\} . \\
& A=\left(\begin{array}{cccccc}
-5 & 0.1 & 1.2 & 0 & 0 & 1 \\
0.1 & -3 & 0 & -0.3 & 0 & -1 \\
1.2 & 0 & -6 & -2 & 0.5 & -2 \\
0 & -0.3 & -2 & -4 & 0.4 & 0.5 \\
0 & 0 & 0.5 & 0.4 & -4 & -0.8 \\
1 & -1 & -2 & 0.5 & -0.8 & -8
\end{array}\right), B=\left(\begin{array}{c}
1 \\
2 \\
1 \\
3 \\
2 \\
0.8
\end{array}\right), C=B^{T}, D=2 .
\end{aligned}
$$

whose inverse is causal and stable. We can compute

$$
\eta=\left\|\left(G_{2}\right)^{-1}\right\|_{H_{\infty}}=0.5556, \rho=0.213 .
$$

The error in the transfer function $G_{1}$ and $G_{2}$ is given by $\gamma=0.0461$. Thus, $\gamma<\rho$ and (13) holds because

$$
\frac{1}{\eta^{2}}-\left(1+2(\rho-\gamma) \frac{1}{\rho}+(\rho-\gamma) \gamma\right)=0.6695>0
$$

From Theorem 2, we can conclude that $G_{1}$ is OSP for $\tilde{\rho}=$ $\rho-\gamma=0.213-0.0461=0.1669$. This is true because the OFP for $G_{1}$ is given by 0.211 , larger than $\tilde{\rho}=0.1669$.

Example 3: The original system $G_{1}$ is given by (19). Its second-order approximation is given by

$$
G_{2}=\frac{2 s^{2}+42.06 s+183.8}{s^{2}+11.22 s+26.79},
$$

which is VSP for $(\rho, \nu)$, where $\nu=1.2, \rho=0.01$. This can be verified through $\Pi \leq 0$ [16], where $\Pi$ is given by

$$
\left[\begin{array}{cc}
A^{T} P+P A+\rho C^{T} C & P B-\left(1 / 2 C^{T}-\rho C^{T} D\right) \\
B^{T} P-\left(1 / 2 C-\rho D^{T} C\right) & \nu I+\rho D^{T} D-D
\end{array}\right],
$$

with $A, B, C, D$ as a minimal realization of $G_{2}$ and $P=I$.

The error in $G_{1}$ and $G_{2}$ is given by $\gamma=0.0042$. For our choice of $\rho, \nu$, we obtain

$$
\nu^{2}-\frac{2(\rho-\gamma)}{\rho}-(\rho-\gamma) \gamma=0.2869>0,
$$

therefore (15) is satisfied. According to Theorem 3, the original system $G_{1}$ is $\operatorname{VSP}$ for $(\tilde{\rho}, \tilde{\nu})$, where

$$
\tilde{\nu}=\nu-\gamma=1.1958, \tilde{\rho}=\rho-\gamma=0.0058 .
$$

This can also be verified through $\Pi \leq 0$ by setting $P=I$ and substituting $\tilde{\rho}, \tilde{\nu}$ for $\rho, \nu$, respectively.

Remark 6: Note that a higher-order reduced model will result in smaller difference in the transfer functions or the passivity levels. To verify Theorem 4, a simple example is presented in [15] (not in this paper due to space limitations).

\section{CONCLUDING REMARKS}

In this paper, we established conditions under which the passivity properties of a system can be obtained by analyzing its approximation. The approximate model is assumed to be input/output/very strictly passive and the general result states that if the error between the system and its approximation is small, the original system has a guaranteed passivity level as well. The analysis is extended to a general case when the approximation is QSR dissipative (not necessarily passive). The results may be interpreted as robustness properties of passivity with respect to model uncertainties. Our results can be used to derive variations in the passivity levels of a linear system and its reduced-order approximation.

\section{REFERENCES}

[1] A. van der Schaft, L2-Gain and Passivity Techniques in Nonlinear Control, 2nd ed. Springer, 2000.

[2] D. Hill and P. Moylan, "The stability of nonlinear dissipative systems," Automatic Control, IEEE Transactions on, vol. 21, no. 5, pp. 708 711, Oct. 1976.

[3] J. Bao and P. L. Lee, Process Control: The passive systems approach, 1st ed. Springer-Verlag, Advances in Industrial Control, London, 2007.

[4] W. S. Levine, Control System Fundamentals, 1st ed. Boca Raton, FL, USA: CRC Press, Inc., 1999.

[5] A. C. Antoulas, Approximation of Large-Scale Dynamical Systems. Society for Industrial and Applied Mathematics, 2005.

[6] H. Nijmeijer, R. Ortega, A. Ruiz, and A. van der Schaft, "On passive systems: from linearity to nonlinearity," in Proceedings 2nd IFAC Symposium on Nonlinear Control Systems (NOLCOS 2004), June 1992, pp. 214-219.

[7] S. Gugercin and A. C. Antoulas, "A survey of model reduction by balanced truncation and some new results," International Journal of Control, vol. 77, no. 8, pp. 748-766, 2004.

[8] J. Phillips, L. Daniel, and L. Silveira, "Guaranteed passive balancing transformations for model order reduction," Computer-Aided Design of Integrated Circuits and Systems, IEEE Transactions on, vol. 22, no. 8, pp. 1027 - 1041, Aug. 2003.

[9] M. McCourt and P. Antsaklis, "Control design for switched systems using passivity indices," in American Control Conference (ACC), 2010, pp. $2499-2504$.

[10] W. Su and L. Xie, "Robust control of nonlinear feedback passive systems," Systems and Control Letters, vol. 28, no. 2, pp. 85 - 93, 1996.

[11] W. Lin and T. Shen, "Robust passivity and feedback design for minimum-phase nonlinear systems with structureal uncertainty," $A u$ tomatica, vol. 35, no. 1, pp. $35-47,1999$.

[12] R. Lozano, B. Brogliato, O.Egeland, and B. Maschke, Dissipative Systems Analysis and Control: Theory and Applications, 1st ed. London: Springer, 2000.

[13] H. K. Khalil, Nonlinear Systems, 3rd ed. Prentice Hall, Upper Saddle River, New Jersey, 2002.

[14] J. C. Willems, "Dissipative dynamical systems part ii: Linear systems with quadratic supply rates," Archive for Rational Mechanics and Analysis, vol. 45, pp. 352-393, 1972.

[15] M. Xia, P. Antsaklis, and V. Gupta, "Passivity and dissipativity of a system and its approximation," ISIS Technical Report, Sep 2012.

[16] N. Kottenstette and P. Antsaklis, "Relationships between positive real, passive dissipative, amp; positive systems," in American Control Conference (ACC), 2010, pp. $409-416$. 